IFUSP /P-1068

\title{
Volume-forms and Minimal Action Principles in Affine Manifolds
}

\author{
Alberto Saa \\ Instituto de Física \\ Universidade de São Paulo, Caixa Postal 20516 \\ 01498-970 São Paulo, Brazil
}

\begin{abstract}
Through the analyses of volume-forms in differentiable manifolds, it is shown that the usual way of defining minimal action principles for field theory on curved spacetimes is not appropriate on non-riemannian manifolds. An alternative approach, based in a new volume-form, is proposed and confronted with the standard one. The new volume element is explicitly used in the study of Einstein-Cartan theory of gravity and its relation to string theory, in connection with some recent results on the subject.
\end{abstract}

Key words: Volume-forms, Minimal Action Principles

PACS: 04.50.+h, 12.10.Gq 


\section{Introduction}

This work discusses the problem of volume definition in differentiable manifolds and its relation with minimal action principles. Action principles are the starting point for several models in Physics, and they usually are formulated in non-euclidean (non-minkowskian) manifolds. In all these models, lagrangians are integrated over the manifold, and under the variational principle hypothesis the dynamical equations are gotten. Whenever such lagrangians are scalars, they are integrated by using a covariant volume elements so that in a given coordinate system one has

$$
S=\int \mathcal{L} d \text { vol }=\int \mathcal{L} \sqrt{|g|} d^{n} x
$$

Usually, the quantity $\sqrt{|g|}$ is introduced with the argument that it makes the euclidean volume element $d^{n} x$ covariant. Typically, the cases where gravitation is incorporated into field theory are described by actions of the type (1). In these cases, space-time can be assumed to be non-riemannian manifolds, as for example in the Einstein-Cartan theory of gravity [1].

It will be shown that, for affine manifolds, there are natural compatibility conditions that a volume element should obey. These conditions will be used in order to construct compatible volume elements for general affine manifolds, and as one of their consequences, one will have that for non-riemannian manifolds the usual volume element (1) is not the more appropriate one. This is a very important point, since (1) is adopted for non-riemannian manifolds as well.

Compatible volume elements demand some restrictions on the non-riemannian structure of the manifold, or in other words, general affine manifolds may not admit compatible volume elements. In these cases, it is not clear how 
to choose a volume element and consequently, how to define action principles for field theory.

In this work, $\mathcal{M}$ is an $n$-dimensional $C^{\infty}$ differentiable oriented manifold, and $\Omega^{m}(\mathcal{M})$ the space of differential $m$-forms on it. $\mathcal{M}$ is called an affine manifold if it is endowed with a linear connection $\Gamma_{\beta \gamma}^{\alpha}$, which is used to define the covariant derivative of tensor valued differential forms

$$
D \Pi_{\beta}^{\alpha}=d \Pi_{\beta}^{\alpha}+\omega_{\rho}^{\alpha} \wedge \Pi_{\beta}^{\rho}-\omega_{\beta}^{\rho} \wedge \Pi_{\rho}^{\alpha}-w \omega \wedge \Pi_{\beta}^{\alpha},
$$

where $\omega_{\beta}^{\alpha}=\Gamma_{\mu \beta}^{\alpha} d x^{\mu}, \omega=\Gamma_{\alpha \mu}^{\alpha} d x^{\mu}$, and $w$ is the weight of $\Pi_{\beta}^{\alpha}$. Of course, (2) can be extended for tensor valued forms of any rank. Considering a $(p, q)$ relative tensor of weight $w, A_{\left(\beta_{1} . . \beta_{p}\right)}^{\left(\alpha_{1} \ldots \alpha_{p}\right)}$, as $p \times q$ differential 0 -forms one gets from (2) the usual formula for the covariant derivative of relative tensors:

$$
\begin{aligned}
& D A_{(\beta)}^{(\alpha)}=\left(D_{\mu} A_{(\beta)}^{(\alpha)}\right) d x^{\mu}= \\
& =\left(\partial_{\mu} A_{(\beta)}^{(\alpha)}+\sum_{i=1}^{p} \Gamma_{\mu \rho}^{\alpha_{i}} A_{(\beta)}^{\left(\alpha_{1} \ldots . . \alpha_{p}\right)}-\sum_{j=1}^{q} \Gamma_{\mu \beta_{j}}^{\rho} A_{\left(\beta_{1} . . . . \beta_{q}\right)}^{(\alpha)}-w \Gamma_{\rho \mu}^{\rho} A_{(\beta)}^{(\alpha)}\right) d x^{\mu} .
\end{aligned}
$$

We will assume also that a metric tensor $g_{\alpha \beta}$ is defined on $\mathcal{M}$ so that

$$
d s^{2}=g_{\alpha \beta} d x^{\alpha} d x^{\beta} .
$$

The anti-symmetrical part of the affine connection

$$
S_{\alpha \beta}^{\gamma}=\frac{1}{2}\left(\Gamma_{\alpha \beta}^{\gamma}-\Gamma_{\beta \alpha}^{\gamma}\right),
$$

defines a new tensor, the torsion tensor $S_{\alpha \beta}^{\gamma}$. It is well known that the $n^{3}$ independent components of the affine connection can be written as function of the metric (田), the torsion (5), and the non-metricity tensor, defined by

$$
N_{\alpha \beta \gamma}=D_{\alpha} g_{\beta \gamma}
$$


The expression for the connection as a function of such quantities is

$$
\Gamma_{\beta \gamma}^{\alpha}=\left\{\begin{array}{c}
\alpha \\
\beta \gamma \gamma
\end{array}\right\}-K_{\beta \gamma}^{\alpha}+V_{\beta \gamma}^{\alpha},
$$

where $\left\{\begin{array}{l}\alpha \\ \beta \gamma\end{array}\right\}$ are the Christoffel symbols, $K_{\alpha \beta}{ }^{\gamma}$ is the contorsion tensor,

$$
K_{\alpha \beta}^{\gamma}=-S_{\alpha \beta}^{\gamma}+S_{\beta}^{\gamma}{ }^{\gamma}-S_{\alpha \beta}^{\gamma},
$$

and $V_{\alpha \beta \gamma}$ is given by:

$$
V_{\alpha \beta \gamma}=\frac{1}{2}\left(N_{\alpha \beta \gamma}-N_{\gamma \alpha \beta}-N_{\beta \gamma \alpha}\right) .
$$

For simplicity, the traces of $S_{\nu \mu}{ }^{\rho}$ and $V_{\nu \mu}{ }^{\rho}$ will be denoted by $S_{\mu}$ and $V_{\mu}$ respectively,

$$
\begin{aligned}
S_{\mu} & =S_{\rho \mu}{ }^{\rho}, \\
V_{\mu} & =V_{\rho \mu}{ }^{\rho} .
\end{aligned}
$$

An affine manifold $\mathcal{M}$ is called a Riemann-Cartan manifold if $N_{\alpha \beta \gamma}=0$, and a riemannian one if $N_{\alpha \beta \gamma}=0$ and $S_{\alpha \beta \gamma}=0$. In all these cases, the connection is said to be metric-compatible.

\section{Volume-forms in Affine Manifolds}

In this section, it will be introduced the notion of compatible volume-form, and it will be derived the necessary conditions that an affine manifold must obey in order to be possible the definition of a compatible volume element on it.

Definition 1. A volume-form on $\mathcal{M}$ is a nowhere vanishing $n$-form $v \in$ $\Omega^{n}(\mathcal{M})$. [3] 
A volume-form, in general, can be constructed by using $n$ linearly independent 1 -forms $\left(\theta^{1} \wedge \ldots \wedge \theta^{n} \neq 0\right)$ and non-vanishing 0 -forms,

$$
v=f \theta^{1} \wedge \ldots \wedge \theta^{n}
$$

We will assume that $f$ is a positive non-vanishing $C^{\infty}$ scalar function. The volume-form (11) defines a volume element on $\mathcal{M}$. If $\left\{\theta^{i}\right\}$ is assumed to be an orthonormal set of 1-forms, one has the following expression for the volume-form in general coordinates $\left\{x^{i}\right\}$

$$
v=f(x) \sqrt{|g|} d x^{1} \wedge \ldots \wedge d x^{n}
$$

as one can check using that if $\Lambda$ is the change of basis matrix $\left(\theta^{i}=\Lambda_{j}^{i} d x^{j}\right)$, then $v=f(x) \operatorname{det}(\Lambda) d x^{1} \wedge \ldots \wedge d x^{n}$. The determinant $\operatorname{det}(\Lambda)$ can be obtained by using the transformation properties of the metric tensor, and that $\left\{\theta^{i}\right\}$ is orthonormal.

Using (12) we have for the total volume of the manifold

$$
\int v=\int f(x) \sqrt{|g|} d x^{1} d x^{2} \ldots d x^{n}=\int d \mathrm{vol}
$$

The usual volume element for riemannian space-times is obtained by choosing $f(x)=1$.

In an affine manifold, one can require certain compatibility conditions between the affine connection and the volume-form. For a differentiable manifold with volume-form $v$, one usually defines the divergence of a vector field $A, \operatorname{div} A$, by [2]

$$
(\operatorname{div} A) v=£_{A} v
$$

where $£_{A}$ is the Lie derivative along the direction $A$. However, if the manifold is endowed with an affine connection, we can define the divergence of a vector 
field in a very natural way by using the covariant derivative,

$$
\operatorname{div}_{\Gamma} A=D_{\mu} A^{\mu}
$$

One can use (14) and (15) to define a criterion of compatibility between the affine connection and the volume-form.

Definition 2. A volume-form $v$ is compatible with the affine connection if

$$
£_{A} v=\left(D_{\mu} A^{\mu}\right) v
$$

for any vector field $A$.

One can check that the riemaniann volume-form $\omega=\sqrt{|g|} d x^{1} \wedge \ldots \wedge d x^{n}$ and the Christoffel symbols are compatible. It can be inferred also that the volume-form $\omega$ is not compatible with the connection for a Riemann-Cartan manifold,

$$
£_{A} \omega=\left(D_{\mu} A^{\mu}-2 S_{\mu} A^{\mu}\right) \omega \neq\left(D_{\mu} A^{\mu}\right) \omega .
$$

The incompatibility of the usual volume-form and the connection for nonriemannian manifolds introduces a new question: Is it possible to define a volume-form compatible with the connection for non-riemannian manifolds? The answer is that sometimes it is, as we will see.

Proposition 1. An affine manifold admits a volume-form compatible with the connection only if the form $\left(V_{\beta}+2 S_{\beta}\right) d x^{\beta}$ is exact.

Proof: In an affine manifold with volume-form $v$ one has

$$
£_{A} v=\left[A^{\mu} D_{\mu}(f(x) \sqrt{|g|})+f(x) \sqrt{|g|} D_{\mu} A^{\mu}\right] d x^{1} \wedge \ldots \wedge d x^{n}
$$

and in order to get (16) for arbitrary $A^{\mu}$ one needs

$$
\begin{aligned}
D_{\mu}(f(x) \sqrt{|g|}) & =\sqrt{|g|} \partial_{\mu} f(x)+f(x) \partial_{\mu} \sqrt{|g|}-\left\{\begin{array}{l}
\rho \\
\rho \mu
\end{array}\right\} f(x) \sqrt{|g|} \\
& -\left(V_{\mu}+2 S_{\mu}\right) f(x) \sqrt{|g|}=0
\end{aligned}
$$


which leads to $\partial_{\mu} \ln f(x)=V_{\mu}+2 S_{\mu}$, or equivalently

$$
V_{\mu} d x^{\mu}+2 S_{\mu} d x^{\mu}=d \ln f(x)
$$

where $d$ stands to exterior derivative.

From (20) the 1-form in question is closed as consequence of Poincare's lemma. If the form $\left(V_{\beta}+2 S_{\beta}\right) d x^{\beta}$ is not closed, the affine manifold does not admit a compatible volume-form. A connection compatible volume-form in an affine manifold will be given by

$$
v=e^{2 \Theta} \sqrt{|g|} d x^{1} \wedge \ldots \wedge d x^{n}
$$

where $\partial_{\mu} \Theta=S_{\mu}+\frac{1}{2} V_{\mu}$, and we also have that

$$
\Gamma_{\rho \mu}^{\rho}=\partial_{\mu} \ln \left(e^{2 \Theta} \sqrt{|g|}\right)
$$

Proposition 2. If an affine manifold is endowed with a volume-form compatible with the connection, one has the following generalized Gauss formula

$$
\int_{\mathcal{M}} D_{\mu} A^{\mu} d \mu=\int_{\partial \mathcal{M}} A^{\mu} d \Sigma_{\mu}
$$

where $d \Sigma_{\mu}$ is the compatible surface element, given by:

$$
d \Sigma_{\mu}=\frac{e^{2 \Theta} \sqrt{|g|}}{(n-1) !} \varepsilon_{\mu \alpha_{2} \alpha_{3} \ldots \alpha_{n}} d x^{\alpha_{2}} \wedge d x^{\alpha_{3}} \ldots \wedge d x^{\alpha_{n}} .
$$

Proof: By choosing a form $\omega=\frac{e^{2 \Theta \sqrt{|g|}}}{(n-1) !} \varepsilon_{\alpha_{1} \alpha_{2} \ldots \alpha_{n}} A^{\alpha_{1}} d x^{\alpha_{2}} \wedge \ldots \wedge d x^{\alpha_{n}}$, using (22) and Stokes' theorem

$$
\int_{\mathcal{M}} d \omega=\int_{\partial \mathcal{M}} \omega
$$

one gets $(23$ 
For riemannian manifolds, the connection compatible volume-form can be obtained by using the Hodge $\left({ }^{*}\right)$ operator. The $\left({ }^{*}\right)$ is a linear operator [3]

$$
{ }^{*}: \Omega^{m}(\mathcal{M}) \rightarrow \Omega^{n-m}(\mathcal{M})
$$

which for a Riemannian manifold has the following action on a basis vector of $\Omega^{m}(\mathcal{M})$

$$
*\left(d x^{\alpha_{1}} \wedge d x^{\alpha_{2}} \wedge \ldots \wedge d x^{\alpha_{m}}\right)=\frac{\sqrt{|g|}}{(n-m) !} \varepsilon_{\beta_{m+1} \ldots \beta_{n}}^{\alpha_{1} \ldots \alpha_{m}} d x^{\beta_{m+1}} \wedge \ldots \wedge d x^{\beta_{n}}
$$

where $\varepsilon_{\alpha_{1} \ldots \alpha_{n}}$ is the totally anti-symmetrical symbol, and $\varepsilon_{\beta_{m+1} \ldots \beta_{n}}^{\alpha_{1} \ldots \alpha_{m}}$ is constructed by using the metric tensor. The action of (27) on the basis vector of $\Omega^{0}(\mathcal{M})$ gives

$$
{ }^{*} 1=\frac{\sqrt{|g|}}{n !} \varepsilon_{\alpha_{1} \ldots \alpha_{n}} d x^{\alpha_{1}} \wedge \ldots \wedge d x^{\alpha_{n}}=\sqrt{|g|} d^{n} x,
$$

which is the compatible volume element for a Riemannian manifold.

Proposition 3. If an affine manifold $\mathcal{M}$ admits a connection compatible volume-form, it can be obtained using the modified Hodge $\left(^{*}\right)$ operator given by

$$
*\left(d x^{\alpha_{1}} \wedge d x^{\alpha_{2}} \wedge \ldots \wedge d x^{\alpha_{m}}\right)=\frac{e^{2 \Theta} \sqrt{|g|}}{(n-m) !} \varepsilon_{\beta_{m+1} \ldots \beta_{n}}^{\alpha_{1} \ldots \alpha_{m}} d x^{\beta_{m+1}} \wedge \ldots \wedge d x^{\beta_{n}} .
$$

Proof: It is clear that the action of (29) in the basis vector for $\Omega^{0}(\mathcal{M})$ gives:

$$
{ }^{*} 1=\frac{e^{2 \Theta} \sqrt{|g|}}{n !} \varepsilon_{\alpha_{1} \ldots \alpha_{n}} d x^{\alpha_{1}} \wedge \ldots \wedge d x^{\alpha_{n}}=e^{2 \Theta} \sqrt{|g|} d^{n} x,
$$

which is the compatible volume-form 


\section{Physical Applications}

In this section, the results of Sect. 2 will be used in the study of EinsteinCartan theory of gravity and its relation with string theory.

In the Einstein-Cartan theory of gravity, the space-time is assumed to be a Riemann-Cartan manifold. It is in accordance with the experimental data and it has also theoretical importance, since it is the theory that arises from the local gauge theory for the Poincaré's group [1]. The dynamical equations for such theory are gotten via a minimal action principle of the type (1), and the lagrangians for external fields are usually obtained from the minkowskian ones by minimal coupling procedure.

We propose that, instead of (11), the action formulation for EinsteinCartan theory shall use the compatible volume element of section 2. Of course the restriction that the space-time manifold admits such volume element (Proposition 1) is implicit. The dynamical equations will be different from the usual ones, and probably a final answer on which is more appropriate can be given only by experimental facts. However, there are theoretical evidences on behalf of the new action, and they will be pointed out in this section.

According to our hypothesis, the Einstein-Cartan gravity equations shall be obtained from a Hilbert-Einstein action using the compatible volume element,

$$
\begin{aligned}
S & =-\int e^{2 \Theta} \sqrt{-g} d^{4} x \mathcal{R} \\
& =-\int e^{2 \Theta} \sqrt{-g} d^{4} x\left(R+4 \partial_{\mu} \Theta \partial^{\mu} \Theta-K_{\nu \rho \alpha} K^{\alpha \nu \rho}\right)+\text { surf. terms }
\end{aligned}
$$

where the generalized Gauss' formula (23) was used. In (31), $\mathcal{R}$ is the scalar of curvature of the Riemann-Cartan manifold, calculated by the contraction 
of the curvature tensor obtained using the full connection, and $R$ is the usual riemannian scalar of curvature, calculated from the Christoffel symbols. The following conventions are adopted: $\operatorname{sign}\left(g_{\mu \nu}\right)=(+,-,-\ldots), R_{\alpha \nu \mu}^{\beta}=$ $\partial_{\alpha} \Gamma_{\nu \mu}^{\beta}+\Gamma_{\alpha \rho}^{\beta} \Gamma_{\nu \mu}^{\rho}-(\alpha \leftrightarrow \nu)$, and $R_{\nu \mu}=R_{\alpha \nu \mu}^{\alpha}$.

The similarity between (31) and the action for the dilaton gravity [6, (7] is surprising. The "torsion potential" $\Theta$ can be identified with the dilaton field, and (31) can provide a geometrical interpretation for the dilaton gravity [8]. Another feature of the proposed action is that, due to the peculiar $\Theta$ dependence of the action (31), the trace of the torsion tensor can propagate, i.e. it can exist non-vanishing solutions for torsion in the vacuum. The torsion mediated interactions are not of contact type anymore. The traceless part of the torsion tensor will be zero in the vacuum, as in the usual EinsteinCartan theory.

Another problem that can be analyzed with the results of Sect. 2 is the description of Maxwell fields on Riemann-Cartan space-times. In order to study Maxwell's equations in a metric differentiable manifold, we introduce a fundamental quantity, the (local) electromagnetic potential 1-form

$$
A=A_{\alpha} d x^{\alpha}
$$

and from the potential 1-form we can define the Faraday's 2-form

$$
F=d A=\frac{1}{2} F_{\alpha \beta} d x^{\alpha} \wedge d x^{\beta},
$$

where $F_{\alpha \beta}=\partial_{\alpha} A_{\beta}-\partial_{\beta} A_{\alpha}$ is the usual electromagnetic tensor.

The homogenous Maxwell's equations arise naturally due to the definition (33) as a consequence of Poincaré's lemma

$$
d F=d(d A)=\frac{1}{2} \partial_{\gamma} F_{\alpha \beta} d x^{\gamma} \wedge d x^{\alpha} \wedge d x^{\beta}=0,
$$


and in terms of components we have

$$
\partial_{[\gamma} F_{\alpha \beta]}=0
$$

where [ ] means antisymmetrization.

The non-homogenous equations in Minkowski space-time are given by

$$
d^{*} F=4 \pi^{*} J
$$

where $J=J_{\alpha} d x^{\alpha}$ is the current 1 -form, and $\left(^{*}\right)$ is the Hodge operator in Minkowski space-time. ${ }^{*} J$ and ${ }^{*} F$ are given by:

$$
\begin{aligned}
{ }^{*} J & =\frac{1}{3 !} \varepsilon_{\alpha \beta \gamma \delta} J^{\alpha} d x^{\beta} \wedge d x^{\gamma} \wedge d x^{\delta}, \\
{ }^{*} F & =\frac{1}{4} \varepsilon_{\alpha \beta \gamma \delta} F^{\gamma \delta} d x^{\alpha} \wedge d x^{\beta} .
\end{aligned}
$$

By an accurate analysis of (36), one can see that it is not general covariant in a curved space-time, because of ${ }^{*} F$ is not a scalar 2 -form, but it is a relative scalar 2 -form with weight -1 , due to the anti-symmetrical symbol. Now we assume that the manifold is endowed with a connection to use it in order to cast (36) in a covariant way. This is done by substituting the exterior derivative by the covariant one

$$
d^{*} F \rightarrow \mathcal{D}^{*} F=\frac{1}{3 !}\left(\partial_{\alpha}{ }^{*} F_{\beta \gamma}+\Gamma_{\rho \alpha}^{\rho}{ }^{*} F_{\beta \gamma}\right) \delta_{\mu \nu \omega}^{\alpha \beta \gamma} d x^{\mu} \wedge d x^{\nu} \wedge d x^{\omega}
$$

where $\delta_{\mu \nu \omega}^{\alpha \beta \gamma}$ is the generalized Kronecker delta. The covariant exterior derivative in (39) takes into account that ${ }^{*} F_{\alpha \beta}=\frac{1}{2} \varepsilon_{\alpha \beta \gamma \delta} F^{\gamma \delta}$ is a relative $(0,2)$ tensor with weight -1 . One can check that $\mathcal{D}^{*} F$ is a relative scalar 3 -form with weight -1 . We have then the following covariant generalization of (36)

$$
\mathcal{D}^{*} F=4 \pi^{*} J
$$


Equations (34) are already in a general covariant form in any differentiable manifold.

Taking the covariant exterior derivative in both sides of (40) we get

$$
4 \pi \mathcal{D}^{*} J=\frac{1}{4 !}\left(\partial_{\lambda} \Gamma_{\rho \mu}^{\rho}\right){ }^{*} F_{\nu \omega} \delta_{\alpha \beta \gamma \delta}^{\lambda \mu \nu \omega} d x^{\alpha} \wedge d x^{\beta} \wedge d x^{\gamma} \wedge d x^{\delta},
$$

and to have a generalized conservation condition for the current we need that

$$
\partial_{\lambda} \Gamma_{\rho \mu}^{\rho}-\partial_{\mu} \Gamma_{\rho \lambda}^{\rho}=0
$$

which has locally as general solution:

$$
\Gamma_{\rho \mu}^{\rho}=\partial_{\mu} f(x)
$$

Using that $\left\{\rho_{\rho \mu}\right\}=\partial_{\mu} \ln \sqrt{-g}$, equation (43) will have general solution only if the trace of the torsion tensor is derivable from a scalar potential, or, that is equivalent, that the space-time admits a compatible volume-form (Proposition 1). In this case $f(x)=\ln \left(e^{2 \Theta} \sqrt{-g}\right)$.

One can ask now if it is possible to obtain the non-homogeneous equations (40) from an action principle. We know that in Minkowski space-time, the non-homogeneous equations are gotten from the following action

$$
S=-\int\left(4 \pi^{*} J \wedge A+\frac{1}{2} F \wedge^{*} F\right) .
$$

The action (44) can be cast in a covariant way by using the modified Hodge $\left({ }^{*}\right)$ operator (Proposition 3 ). In this case one gets the following coordinate expression for the generally covariant generalization of (44)

$$
S=\int d^{4} x e^{2 \Theta} \sqrt{-g}\left(-\frac{1}{4} F_{\alpha \beta} F^{\alpha \beta}+4 \pi J^{\alpha} A_{\alpha}\right) .
$$

It is easy to check that we can obtain equations (40) from the action (45). Under (43), the covariant equation (40) can be obtained directly from (36) 
by using the modified Hodge operator. We can check also that equations (35) and (40) are invariant under the usual $U(1)$ gauge transformation

$$
A_{\mu} \rightarrow A_{\mu}+\partial_{\mu} \varphi
$$

We would like to stress the importance of the generalized conservation condition to guarantee the gauge invariance of the action (45).

The use of the compatible volume element have brought two main modifications to the problem of Maxwell fields on Riemann-Cartan space-times. First, it is clear from (45) that gauge fields can interact with torsion without destroying gauge invariance, and second, there is no difference if one starts from the action formulation or from the equations of motion. It is important to stress also that the necessary condition to a Riemann-Cartan manifold admit a compatible volume element arises as an "integrability" condition for Maxwell's equations on Riemann-Cartan manifolds.

\section{Final Remarks}

The consequences of using compatible volume elements for minimal action principles for field theory is now under investigation. For the Einstein-Cartan theory, it is already known that the new volume element will modify the dynamical equations for the space-time geometry. Propagation of torsion will be possible, and not only fermion fields will interact with the non-riemannian structure of space-time. The theory become more consistent, since we get the same results starting from the action formulation or from the equations of motion. It is very interesting that the necessary condition of Proposition 2 arises as integrability condition for the matter field equations in RiemannCartan space-times [4, 5]. The compatible volume element may also provide 
a geometrical interpretation for the dilaton gravity, which comes from string theory.

\section{Acknowledgements}

The author is grateful to Josif Frenkel and José Carlos Brunelli. This work was supported by Fundação de Amparo à Pesquisa do Estado de São Paulo. 


\section{References}

[1] F.W. Hehl, P. von der Heyde, G.D. Kerlick, and J.M. Nester, General Relativity with Spin and Torsion: Foundations and Prospects, Rev. Mod. Phys. 48 (1976) 393.

[2] S. Kobayashi and K. Nomizu, Foundations of Differential Geometry, vol. 1, John Willey, New York, 1963.

[3] M. Nakahara, Geometry, Topology and Physics, Adam Hilger, Bristol, 1990.

[4] A. Saa, On Minimal Coupling in Riemann-Cartan Space-times, to be published in Mod.Phys.Lett. A.

[5] A. Saa, Gauge Fields on Riemann-Cartan Space-times, to be published in Int.J.Mod.Phys. A.

[6] C.G. Callan, D. Friedan, E.J. Martinec, and M.J. Perry, Strings in Background Fields, Nucl.Phys. B262 (1985) 593.

[7] M.B. Green, J.H. Schwarz, and E. Witten, Superstring Theory, sect. 3.4, Cambridge University Press, 1987.

[8] A. Saa, Strings in Background Fields and Einstein-Cartan theory of Gravity, Pre-print IFUSP/P-1066 (hep-th/9307095), to be published. 